\title{
A New Approach to Fight Alzheimer's
}

A protein expressed on the surface of neurons may naturally prevent the formation and growth of the amyloid plaques associated with Alzheimer's disease (AD), potentially providing an alternative means to prevent the onset of this devastating neurodegenerative disorder.

$\mathrm{AD}$ affects nearly 2 million people across the United States, and roughly $10 \%$ of people over the age of 65 . In $\mathrm{AD}$ extensive plaques and fibrillary tangles form throughout the brain, with subsequent extensive neuronal deterioration. Although specific details of the pathology of $\mathrm{AD}$ remain controversial, researchers believe that these plaques, composed primarily of a protein known as $\beta$-amyloid, function significantly in subsequent neurodegeneration.

Neprilysin (Nep), a protease expressed on the surface of neurons throughout the brain, is putatively involved in the degradation of a variety of signaling peptides; however, recent research has shown an inverse correlation between Nep activity and the density of amyloid plaques, suggesting that Nep may help to counter the development of these plaques.

Researchers at the Salk Institute (La Jolla, CA) and the University of California, San Diego created a modified lentivirus expressing Nep, and then introduced it into the brains of mice expressing mutant human $\beta$-amyloid so as to determine the effects on plaque formation. They found that amyloid plaques within the injected hemisphere were considerably smaller than those on the untreated side, and that these smaller plaques co-localized with sites of expression of neprilysin (J. Neurosci., 15 March). Furthermore, initial pathological studies suggested that the presence of Nep lentivirus considerably reduced the extent of neurodegeneration, as determined by immunohistochemistry.

These findings suggest an interesting target for future therapeutics, using a protein that apparently degrades plaques or prevents their formation. "People have been looking at the enzymes involved in the initial synthesis and processing of the amyloid," says author Fred Gage. "A new look at the proteins involved in the degradation of amyloid plaques may provide a more general approach to the study of Alzheimer's disease."

According to Gage, the next step will involve studies conducted in live animals to determine the gross phenotypic effects of neprilysin overexpression, and whether his group's lentivirus construct can counteract the behavioral and neurological effects of $\mathrm{AD}$. The researchers also hope that these studies will further illuminate neprilysin's mode of actionwhether it acts to prevent plaque assembly or targets amyloid clumps that are already in place. - M.E.

basis of this developmental barrier, using the UniGene database of murine cDNA libaries to assemble a list of candidate genes (Development, 15 April). They identified 66 genes that were apparently expressed in blastocysts and embryonic germline cells, but not in mature somatic cells; they chose 10 of these for further study on the basis of their temporal expression patterns, which resembled that of Oct4, a known mediator of embryonic stem (ES) cell pluripotency.

Jaenisch and his colleagues studied the expression profiles of these genes in clones derived from both ES and somatic donor cells. For somatic-derived clones, roughly $25 \%$ arrested before reaching the blastocyst stage, and none of these clones showed complete expression of all 11 Oct4-related genes. In addition, nearly $40 \%$ of the blastocyst clones lacked proper expression of at least one of the 11 genes. In contrast, all ESderived clones reached blastocyst stage and showed appropriate gene expression for each of the Oct4-related genes. These clones also displayed proper temporal regulation of these genes, similar to the expression profiles seen in conventional fertilized zygotes.

The authors suggest that the genes identified by this in silico approach may provide a valuable starting point for the comprehensive analysis of the basis of somatic clone nonviability, and for the untangling of the larger networks of gene regulation involved in successful zygotic development.

- M.E.
Hosting a meeting, conference, or workshop? Send listings to:

Lab Animal

345 Park Ave. South

New York, NY 10010-1707

email: labanimal@natureny.com 